\title{
Double-Pass System (Optical Quality Analysis System) for Analysis of the Multifocal Function of a Diffractive Multifocal Intraocular Lens (Acrysof ReSTOR®) Compared to a Monofocal Intraocular Lens (Acrysof IQ®)
}

\author{
Ho Sik Hwang ${ }^{1}$, Hye Young Shin ${ }^{2}$, and Choun-Ki Joo ${ }^{2 *}$ \\ ${ }^{1}$ Department of Ophthalmology, Hallym University, Chuncheon 200-704, Korea \\ ${ }^{2}$ Department of Ophthalmology, Catholic University of Korea, Seoul 137-040, Korea
}

(Received September 3, 2013 : revised February 25, 2014 : accepted February 28, 2014)

\begin{abstract}
In this study, we objectively determined whether the ReSTOR as a multifocal IOL (intraocular lens) has a multifocal function compared to the IQ as a monofocal IOL in vivo by OQAS (Optical Quality Analysis System). Eighteen patients who had cataract surgery with implantation of ReSTOR (27 eyes) and 15 patients with IQ (21 eyes), were included inthis study. Uncorrected distance visual acuity (UCDVA), corrected distance visual acuity (CDVA), uncorrected near visual acuity (UCNVA)and distance corrected near visual acuity (DCNVA) were measured. After setting the artificial pupil size to $3 \mathrm{~mm}$, we performed 'Optical quality'. We inputted defocusdiopters of (objective spherical refraction)(far), (objective spherical refraction-1.25 D)(intermediate),(objective spherical refraction-2.5 D)(near), and (objective spherical refraction-3.5 D)(very near) into 'selected spherical refraction' simulating theoptical quality at far, intermediate, and near distance. We changed the pupil size to $5 \mathrm{~mm}$ and repeated thesame measurements. The UCDVA and CDVA did not show significant differences between the 2 groups. But, theUCNVA and DCNVA of the ReSTOR group were better than those of the IQ group $(p=0.000, p=0.000)$. For $3 \mathrm{~mm}$ pupil, at far distance, modulation transfer function (MTF) cut off and point spread function (PSF) width at $50 \%$ of ReSTOR were worse than those of IQ $(p=0.039, p=0.020)$. At intermediate distance, MTF cut off, Strehl ratio and PSF width at $50 \%$ of ReSTOR were worse than those of IQ $(\mathrm{p}=0.001, \mathrm{p}=0.001, \mathrm{p}=0.000)$. At near distance, MTF cut off of ReSTOR was worse than that of IQ $(p=0.033)$. At very near distance, MTF cut off and Strehl ratio of ReSTOR were worse than those of IQ $(p=0.002, p=0.002)$, but PSF width at $50 \%$ of ReSTOR was better than that of IQ. For $5 \mathrm{~mm}$ pupil, most parameters at each distance, there was no significant difference between the 2 groups. Only PSF width at $50 \%$ of ReSTOR were worse than those of IQ at intermediate distance $(p=0.013)$. It was impossible to show the multifocal function of ReSTOR compared to the IQ byOQAS.
\end{abstract}

Keywords : Cataract, Diffractive, Double-pass system, Intraocular lens, Multifocal

OCIS codes : (050.1965) Diffractive lenses; (170.4460) Ophthalmic optics and devices; (170.4470) Ophthalmology; (330.7328) Visual optics, ophthalmic appliances; (330.7335) Visual optics, refractive surgery

\section{INTRODUCTION}

Recently, the use of multifocal intraocular lenses (IOLs) in cataract surgery has been increasing. As one of the multifocal IOLs, Acrysof ReSTOR ${ }^{\circledR}$ (Alcon Laboratories, Fort Worth, TX) is a diffractive multifocal IOL. It has a multifocal function created by adding diffractive rings to
Acrysof IQ $^{\circledR}$ (Alcon Laboratories). Several studies have evaluated the multifocal function of the ReSTOR by measuring the distance visual acuity and near visual acuity of patients who had cataract surgery with implantation of the lens.[1-6] However, they have a weakness in that they depend on the subjective visual acuity of the patients. Recently, Kim et al reported on the testing of the multifocal

\footnotetext{
*Corresponding author: ckjoo@catholic.ac.kr

Color versions of one or more of the figures in this paper are available online.
} 
function of multifocal IOLs including ReSTOR using an optical bench system [7]. Their results were objective but had a limitation since it was not an in vivo study. Therefore, a study that can objectively measure the multifocal function of multifocal IOLs in vivo is necessary.

The Optical Quality Analysis System (OQAS, Visiometrics S. L., Terrassa, Barcelona, Spain) records the image of a point source after reflection on the retina and a double pass through the ocular media. Details about this system were well described in Vilaseca's studies [8,9]. This equipment can measure the optical quality objectively.

In this study, we measured and compared the optical quality at far and near distances for patients who had cataract surgeries with the implantation of ReSTOR and IQ using this equipment. Thus, the aim of this paper was to objectively determine whether the ReSTOR as a multifocal IOL has multifocal function compared to the IQ as a monofocal IOL in vivo by OQAS.

\section{METHODS}

Eighteen patients who had cataract surgery withimplantation of ReSTOR (27 eyes) and 15 patients with IQ (21 eyes), were included inthis study. The patients who had no intraoperative complications and at least 3 months had passed after the operation were included in this study. The inclusion criteria are IOLpower 18.00 to 25.00 diopters, postoperative corrected distance visual acuity (CDVA) of $20 / 25$ or better, and well-positioned IOL postoperatively. The exclusion criteria are opacity of the ocular media including posterior capsular opacity, corneal surface problems, retinal disease and $5 \mathrm{~mm}$ or smaller pupil after dilatation, and systemic disease that could interfere with the final results (eg, diabetes). We explained the purpose of this study and measurement methods to the patients. We received informed consent from the subjects. The study followed the principles of the Declaration of Helsinki, and the Institutional Review Board of Seoul St. Mary's Hospital (Seoul, Korea) approved the study.

Cataract surgeries were performed by one surgeon (CKJ). The axial length was measured with the IOL Master optical biometer ver. 5 (Carl-Zeiss, Jena, Germany) and corneal power was measured with an auto-keratorefractometer (Canon RK-5; Canon, Tokyo, Japan). The IOL power was selected for emmetropia target with the SRK-2 or SRK-T formula.

Postoperatively, the intraocular pressure (IOP) was measured by noncontact tonometer and refractive error was measured by an auto-keratorefractometer. Uncorrected distance visual acuity (UCDVA) and corrected distance visual acuity (CDVA) of the patients were measured with a 5 m LogMAR (Log of Minimal Angular Resolution) visual acuity chart. In addition, uncorrected near visual acuity (UCNVA) and distance corrected near visual acuity (DCNVA) of the patients were measured by a $40 \mathrm{~cm}$ ETDRS (Early Treatment Diabetic Retinopathy Study) visual acuity chart
(Precision Vision, La Salle, IL). The $40 \mathrm{~cm}$ distance corresponds to the $+2.5 \mathrm{D}$ addition at the spectacle plane.

After visual acuity measurements, the pupils of the patients were dilated with mydriatic eyedrops to simulate pupil size effect on optical quality. Mydrin-P (Santen, Osaka, Japan) was applied to the patients 2 times at 5-minute intervals for dilatation. After 30 minutes, the pupil size and posterior capsule were checked by slit lamp examination.

Figure 1 shows a schematicdiagram of this doublepasssystem $[8,9]$. The instrumentrecordsthe retinal image correspondingto a point source object in near-infraredlight, consisting of a laserdiode $(\lambda=780 \mathrm{~nm})$ coupledto an optical fiber, after reflectionon the retina and a doublepass through the ocular media.A motorized optometer,consisting of two lenses (L3, L4) and two mirrors (M2, M3), was used to measurethe patient's defocus correction. An infrared videocamera $(\mathrm{CCD} 1)$ recorded thedouble-pass images after the light was reflected on theretina and on a beam splitter (BS2). Pupil alignmentwas controlled with an additional camera (CCD2). The entrancepupil had a fixed diameter of $2 \mathrm{~mm}$. The instrument hadan artificial and variable exit pupilwhose image was formed on thepatient's natural pupil plane. Using therecorded images, the point spread function (PSF) and the modulation transferfunction (MTF) were obtained. 'PSF width at $50 \%$ ' is the width of the PSF at

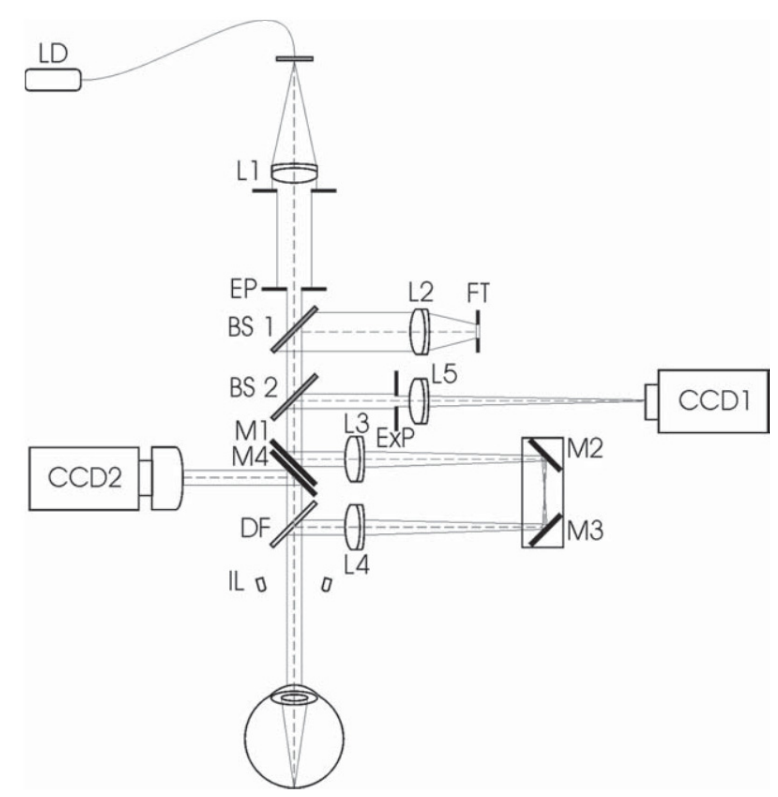

FIG. 1. The double-pass experimental setup (Optical Quality Analysis System [OQAS, VisiometricsS.L.]). LD = laser diode; L1, L2, L3, L4, and L5 = lenses; EP = entrance pupil; $\mathrm{ExP}=$ exit pupil; $\mathrm{BS} 1$ and $\mathrm{BS} 2=$ beam splitter 1 and $2 ; \mathrm{FT}=$ fixation test; $\mathrm{CCD} 1$ and $\mathrm{CCD} 2=\mathrm{CCD}$ cameras 1 and 2; $\mathrm{M} 1, \mathrm{M} 2, \mathrm{M} 3$, and $\mathrm{M} 4=$ mirrors; $\mathrm{DF}=$ dichroic filter; $\mathrm{IL}=$ infrared LEDs (From M. Vilaseca, A. Padilla, J. Pujol, J. C. Ondategui, P. Artal, J. L. Guell: Optical quality one month after verisyse and Veriflex phakic IOL implantation and Zeiss MEL 80 LASIK for myopia from 5.00 to 16.50 diopters, J Refract Surg 2009; 25(8):689-98). 
$50 \%$ of the maximum height. The narrower the width is, the better the optical quality is. MTF cut-off value (cycles/ degree) is the frequency at $1 \%$ of its maximum value. The higher the MTF cut-off value is, the better the optical quality is. The Strehl ratio is thearea below the MTF function weighted with respect to theideal eye. The greater the ratio is, the better the optical quality is.

Optical quality was measured by OQAS as following. The cylindrical refractive error was measured by an autokeratorefractometer. A corresponding cylinder lens was inserted to a lens frame of the OQAS thus the cylindrical refractive error of the eyes were corrected. The patients were instructed to look at the fixation target. After setting the artificial pupil size of the OQAS to $4 \mathrm{~mm}$ (default), we performed 'Objective refraction' and found thespherical error. After changing the artificial pupil size to $3 \mathrm{~mm}$ for 'small pupil' simulation, we performed 'Optical quality'. At this moment,we input defocus diopters of (objective spherical refraction)(far), (objective spherical refraction-1.25 D)(intermediate), and (objective spherical refraction-2.5 D)(near) into 'selected spherical refraction' simulating the optical quality at far, intermediate, and near distances. Additionally, for some patients, we added a defocus diopter $-3.5 \mathrm{D}$ for very near distance. We recorded the MTF cut-off value, Strehl ratio, and PSF width $50 \%$ as the results of 'Optical quality'.Finally, we performed 'IOL accommodation curve' and acquiredthe pseudo-accommodation curve for 12 eyes of ReSTOR and 6 eyes of IQ. By making defocus from $-0.5 \mathrm{D}$ to $+3.5 \mathrm{D}, 18$ serial PSFs with the defocus were obtained. From PSF, the image quality with that defocus was computed by OQAS. Image quality at 0 defocus simulates optical qualityat far, and image quality at $+2.5 \mathrm{D}$ simulates optical qualityat $40 \mathrm{~cm}$. We can assess the multifocal function of ReSTOR by checking the second peaks of the curvesaround $+2.5 \mathrm{D}$. We changed the artificial pupil size to $5 \mathrm{~mm}$ for 'large pupil' simulationand repeated the same measurements. OQAS measurements were performed by an experienced optometrist (CWP).

We found the mean values of the MTF cut-off, Strehl ratio, and width $50 \%$ of the ReSTOR and IQ groups and compared them. For non-parametric statistics, we performed the Mann Whitney u-test. Analyses were done with the statistical software, SPSS version 15 (SPSS Inc. Chicago, IL, USA). In all analyses, a $p$ value $<0.05$ was considered statistically significant.

\section{RESULTS}

Twenty seven eyes from 18 patients (6 males and 12 females) in the ReSTOR group and 21 eyes from 15 patients ( 3 males and 12 females) in the IQ group were included in this study. The mean age of the ReSTOR group was 58.7 years and that of the IQ group was 64.4 years $(p=0.017)$. The mean follow up periods of ReSTOR group and IQ group were 15.6 months and 15.2 months, respectively. Postoperative IOP, spherical equivalent, and astigmatism did not show a significant difference between the 2 groups (Table 1). The UCDVA and CDVA did not show any significant differences between the 2 groups.

TABLE 1. Characteristics of the population in this study

\begin{tabular}{c|c|c|c}
\hline \hline & ReSTOR group & IQ group & $p$-value \\
\hline Eyes/patients & $27 / 18$ & $21 / 15$ & \\
Male : Female & $6: 12$ & $3: 12$ & \\
\hline Age (years) & Mean \pm Standard deviation & Mean \pm Standard deviation & $0.017^{*}$ \\
Follow up (months) & $58.7 \pm 9.2$ & $64.4 \pm 7.4$ & 0.842 \\
Intraocular pressure (mmHg) & $15.6 \pm 4.7$ & $15.2 \pm 3.0$ & 0.638 \\
\hline Postoperative Spherical Equivalent $\left(\mathrm{D}^{\dagger}\right)$ & $11.1 \pm 3.6$ & $11.3 \pm 2.8$ & 0.331 \\
Postoperative Astigmatism $\left(\mathrm{D}^{\dagger}\right)$ & $-0.044 \pm 0.526$ & $-0.208 \pm 0.446$ & 0.491 \\
\hline Postoperative UCDVA & $-0.741 \pm 0.539$ & $-0.607 \pm 0.302$ & 0.243 \\
Postoperative CDVA & $0.073 \pm 0.076$ & $0.056 \pm 0.092$ & 0.377 \\
Postoperative UCNVA & $0.033 \pm 0.055$ & $0.019 \pm 0.039$ & $0.000^{*}$ \\
Postoperative DCNVA $^{\ddagger}$ & $0.148 \pm 0.150$ & $0.414 \pm 0.182$ & $0.000^{*}$ \\
\hline
\end{tabular}

\footnotetext{
${ }^{\dagger} \mathrm{D}$ : Diopters

${ }^{\ddagger}$ LogMAR (Log of Minimal Angular Resolution) unit

UCDVA: Uncorrected distance visual acuity

CDVA: Corrected distance visual acuity

UCNVA: Uncorrected near visual acuity

DCNVA: Distance corrected near visual acuity

${ }^{*} p<0.05$ by Mann-Whitney U-test
} 
TABLE 2. Optical quality measured with OQAS for $3 \mathrm{~mm}$ pupil

\begin{tabular}{|c|c|c|c|c|c|c|}
\hline & & \multicolumn{2}{|c|}{ ReSTOR } & \multicolumn{2}{|c|}{ IQ } & \multirow[b]{2}{*}{$p$-value } \\
\hline & & Number of eyes & $\begin{array}{c}\text { Mean } \pm \text { Standard } \\
\text { deviation }\end{array}$ & Number of eyes & $\begin{array}{c}\text { Mean } \pm \text { Standard } \\
\text { deviation }\end{array}$ & \\
\hline \multirow{3}{*}{$\begin{array}{l}\text { Distant } \\
\left(\begin{array}{ll}0 & \mathrm{D}\end{array}\right)\end{array}$} & MTF cut off (cycles/degree) & 27 & $27.274 \pm 11.404$ & 21 & $33.994 \pm 11.193$ & $0.039^{*}$ \\
\hline & Strehl ratio & 27 & $0.158 \pm 0.083$ & 21 & $0.162 \pm 0.047$ & 0.212 \\
\hline & PSF width at $50 \%$ & 27 & $4.835 \pm 2.035$ & 21 & $3.644 \pm 1.197$ & $0.020^{*}$ \\
\hline \multirow{3}{*}{$\begin{array}{l}\text { Intermediate } \\
(-1.25 \text { D) }\end{array}$} & MTF cut off (cycles/degree) & 27 & $10.323 \pm 3.545$ & 21 & $13.321 \pm 3.027$ & $0.001^{*}$ \\
\hline & Strehl ratio & 27 & $0.080 \pm 0.016$ & 21 & $0.094 \pm 0.017$ & $0.001^{*}$ \\
\hline & PSF width at $50 \%$ & 27 & $10.939 \pm 2.808$ & 21 & $7.895 \pm 1.765$ & $0.000^{*}$ \\
\hline \multirow{3}{*}{$\begin{array}{c}\text { Near } \\
(-2.5 \mathrm{D})\end{array}$} & MTF cut off (cycles/degree) & 27 & $5.018 \pm 1.394$ & 21 & $7.637 \pm 6.429$ & $0.033^{*}$ \\
\hline & Strehl ratio & 27 & $0.054 \pm 0.005$ & 21 & $0.058 \pm 0.030$ & 0.118 \\
\hline & PSF width at $50 \%$ & 27 & $15.983 \pm 4.811$ & 21 & $16.488 \pm 4.707$ & 0.678 \\
\hline \multirow{3}{*}{$\begin{array}{l}\text { Very near } \\
\left(\begin{array}{ll}-3.5 & \mathrm{D})\end{array}\right.\end{array}$} & MTF cut off (cycles/degree) & 18 & $3.995 \pm 1.438$ & 11 & $4.3888 \pm 0.170$ & $0.002^{*}$ \\
\hline & Strehl ratio & 18 & $0.049 \pm 0.005$ & 11 & $0.052 \pm 0.001$ & $0.002^{*}$ \\
\hline & PSF width at $50 \%$ & 18 & $17.295 \pm 8.066$ & 11 & $25.725 \pm 2.632$ & $0.001^{*}$ \\
\hline
\end{tabular}

D: Diopters

MTF: Modulation transfer function

PSF: Point spread function

${ }^{*} p<0.05$ by Mann-Whitney U-test

TABLE 3. Optical quality measured with OQAS for $5 \mathrm{~mm}$ pupil

\begin{tabular}{|c|c|c|c|c|c|c|}
\hline & & \multicolumn{2}{|c|}{ ReSTOR } & \multicolumn{2}{|c|}{ IQ } & \multirow[b]{2}{*}{ p-value } \\
\hline & & $\begin{array}{l}\text { Number } \\
\text { of eyes }\end{array}$ & $\begin{array}{c}\text { Mean } \pm \text { Standard } \\
\text { deviation }\end{array}$ & $\begin{array}{l}\text { Number } \\
\text { of eyes }\end{array}$ & $\begin{array}{c}\text { Mean } \pm \text { Standard } \\
\text { deviation }\end{array}$ & \\
\hline \multirow{3}{*}{$\begin{array}{c}\text { Distant } \\
\left(\begin{array}{ll}0 & \mathrm{D}\end{array}\right)\end{array}$} & MTFcut off (cycles/degree) & 27 & $23.762 \pm 11.867$ & 21 & $25.086 \pm 9.297$ & 0.377 \\
\hline & Strehl ratio & 27 & $0.131 \pm 0.076$ & 21 & $0.132 \pm 0.043$ & 0.350 \\
\hline & PSF width at $50 \%$ & 27 & $5.758 \pm 2.665$ & 21 & $4.900 \pm 2.452$ & 0.205 \\
\hline \multirow{3}{*}{$\begin{array}{l}\text { Intermediate } \\
\left(\begin{array}{ll}-1.25 & \mathrm{D}\end{array}\right)\end{array}$} & MTFcut off (cycles/degree) & 27 & $9.199 \pm 5.357$ & 21 & $10.031 \pm 4.227$ & 0.240 \\
\hline & Strehl ratio & 27 & $0.078 \pm 0.033$ & 21 & $0.079 \pm 0.020$ & 0.334 \\
\hline & PSF width at $50 \%$ & 27 & $14.589 \pm 4.061$ & 21 & $11.757 \pm 3.686$ & $0.013^{*}$ \\
\hline \multirow{3}{*}{$\begin{array}{c}\text { Near } \\
(-2.5 \mathrm{D})\end{array}$} & MTFcut off (cycles/degree) & 27 & $4.495 \pm 1.015$ & 21 & $4.524 \pm 0.430$ & 0.366 \\
\hline & Strehl ratio & 27 & $0.052 \pm 0.003$ & 21 & $0.052 \pm 0.002$ & 0.530 \\
\hline & PSF width at $50 \%$ & 27 & $26.058 \pm 6.643$ & 21 & $26.415 \pm 4.434$ & 0.716 \\
\hline \multirow{3}{*}{$\begin{array}{l}\text { Very near } \\
(-3.5 \quad \mathrm{D})\end{array}$} & MTFcut off (cycles/degree) & 18 & $3.546 \pm 0.605$ & 11 & $3.815 \pm 0.391$ & 0.159 \\
\hline & Strehl ratio & 18 & $0.048 \pm 0.002$ & 11 & $0.049 \pm 0.001$ & 0.146 \\
\hline & PSF width at $50 \%$ & 18 & $29.585 \pm 13.278$ & 11 & $38.495 \pm 9.481$ & 0.061 \\
\hline
\end{tabular}

MTF: Modulation transfer function

PSF: Point spread function

${ }^{*} p<0.05$ by Mann-Whitney U-test

But, the UCNVA of the ReSTOR group (0.148 LogMAR) was better than that of the IQ group $(0.414 \operatorname{LogMAR})(p=$ $0.000)$. The DCNVA of the ReSTOR group $(0.140$ LogMAR) was better than that of the IQ group $(0.457$ LogMAR) $(p=0.000)$.

Table 2 presents the results for the $3 \mathrm{~mm}$ pupil. For far distance $\left(\begin{array}{ll}0 & \mathrm{D}\end{array}\right)$, MTF cut off and PSF width at $50 \%$ of ReSTOR were worse than those of IQ $(p=0.039, p=0.020$, respectively). For intermediate distance $(-1.25 \mathrm{D})$, MTF cut off, Strehl ratio and PSF width at $50 \%$ of ReSTOR were worse than those of $\mathrm{IQ}(p=0.001, p=0.001, p=0.000$, respectively). For near distance $(-2.5 \mathrm{D}), \mathrm{MTF}$ cut off of
ReSTOR was worse than that of IQ $(p=0.033)$. For very near distance $(-3.5 \mathrm{D})$, MTF cut off and Strehl ratio of ReSTOR were worse than those of IQ $(p=0.002, p=0.002)$, but PSF width at $50 \%$ of ReSTOR was better than that of IQ $(p=0.001)$. Table 3 presents the results for the $5 \mathrm{~mm}$ pupil. For most parameters at each distance, there was no significant difference between the 2 groups. Only PSF width at $50 \%$ of ReSTOR wereworsethan those of IQ at intermediate distance $(-1.25 \mathrm{D})(p=0.013)$. Figure 2 are graphs of MTF cut off, Strehl ratio and PSF width at 50\% at each distance using the results of Table 2 and Table 3. 


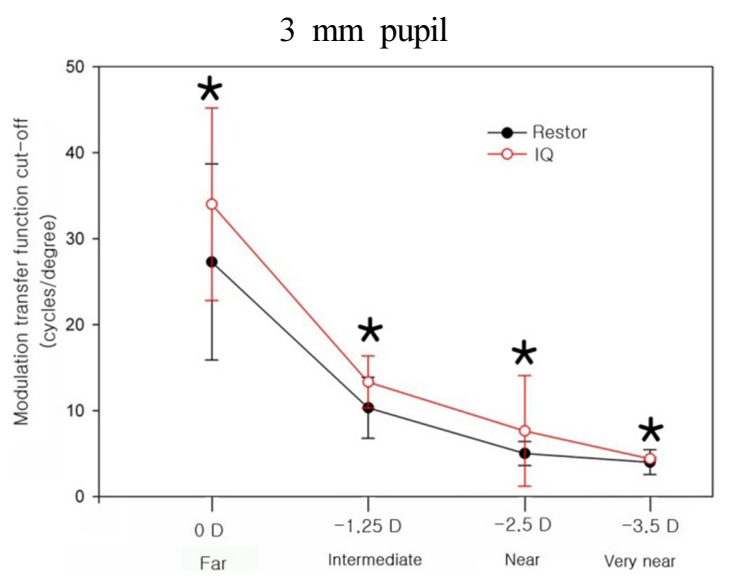

(a)

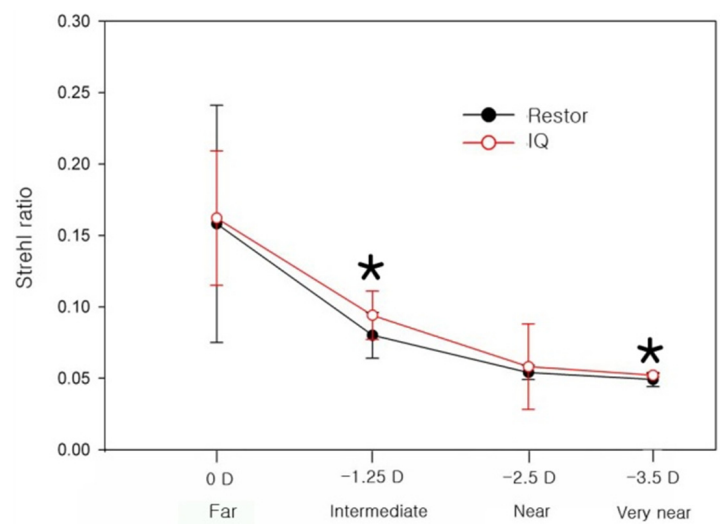

(c)

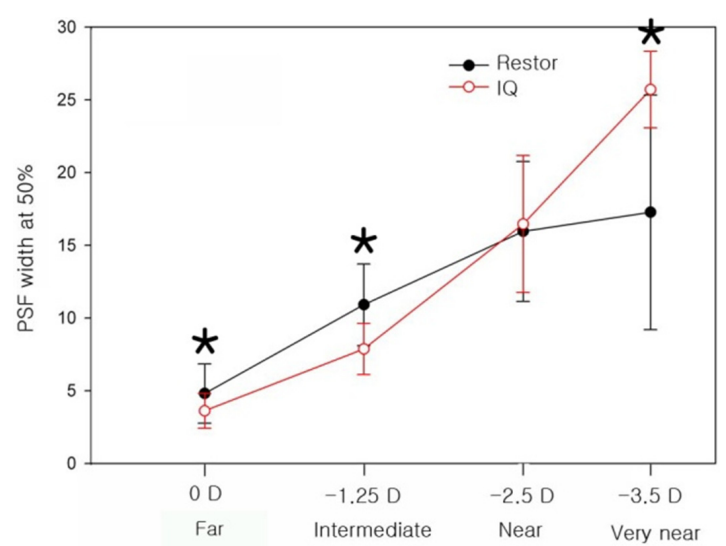

(e)

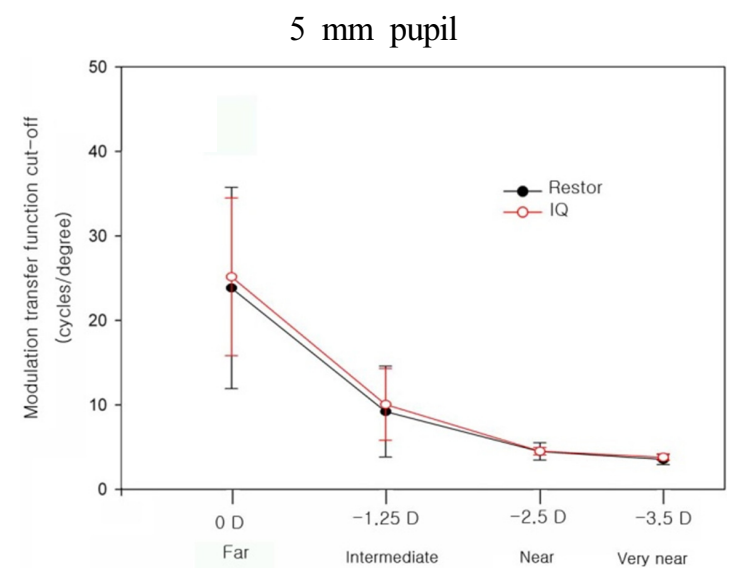

(b)

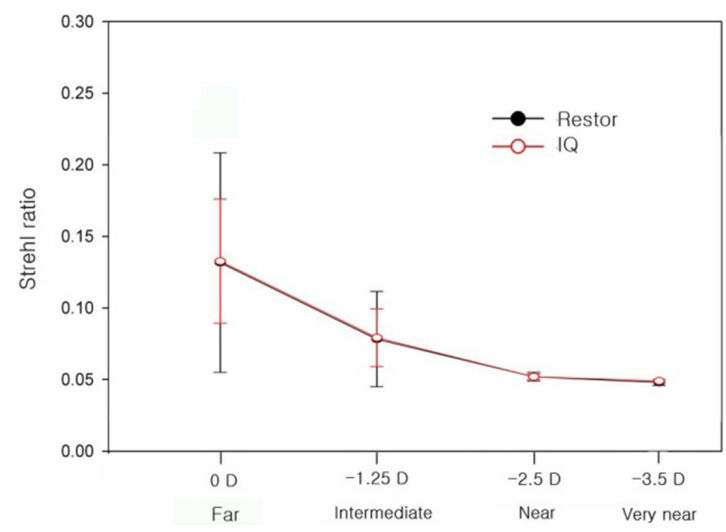

(d)

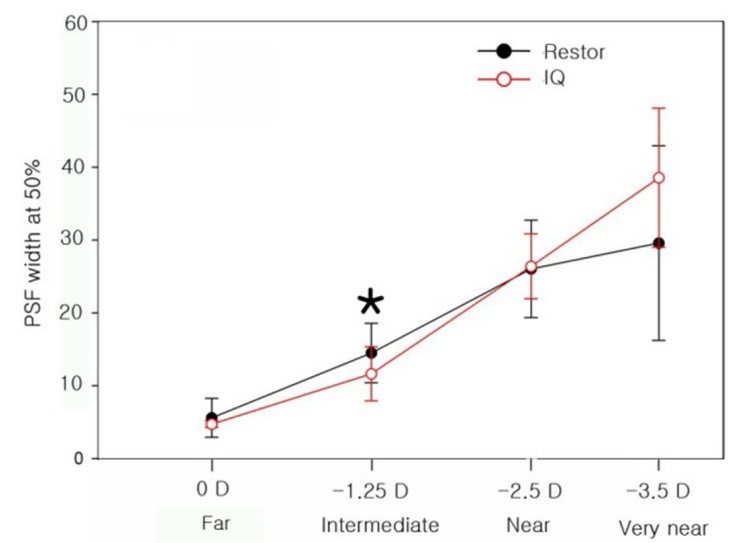

(f)

FIG. 2. Modulation transfer function (MTF) cut off, Strehl ratio and point spread function (PSF) width at $50 \%$ of ReSTOR and IQ lens. (a) MTF cut off, pupil size $3.0 \mathrm{~mm}$; (b) MTF cut off, pupil size $5.0 \mathrm{~mm}$; (c) Strehl ratio, pupil size $3.0 \mathrm{~mm}$; (d) Strehl ratio, pupil size $5.0 \mathrm{~mm}$; (e) PSF width at 50\%, pupil size $3.0 \mathrm{~mm}$; (f) PSF width at 50\%, pupil size $5.0 \mathrm{~mm}$.

$* p<0.05$ by Mann-Whitney U-test

\section{1. $3 \mathrm{~mm}$ Pupil}

With respect to MTF cut off, the optical quality of ReSTOR consistently decreased from $0 \mathrm{D}$ to $-3.5 \mathrm{D}$ without reversal (Fig. 2(a)). At all distance, the optical quality of ReSTOR was worse than that of IQ. With respect to Strehl ratio, the optical quality of ReSTOR also decreased from $0 \mathrm{D}$ to $-3.5 \mathrm{D}$ (Fig. 2(c)). At intermediate and very near distance, the optical quality of ReSTOR was worse than that of IQ. With respect to PSF width at $50 \%$, the optical quality of ReSTOR decreased (increasing PSF width at $50 \%$ )from $0 \mathrm{D}$ to $-3.5 \mathrm{D}$ (Fig. 2(e)). The optical quality of ReSTOR was worse than that of IQ lens at far 


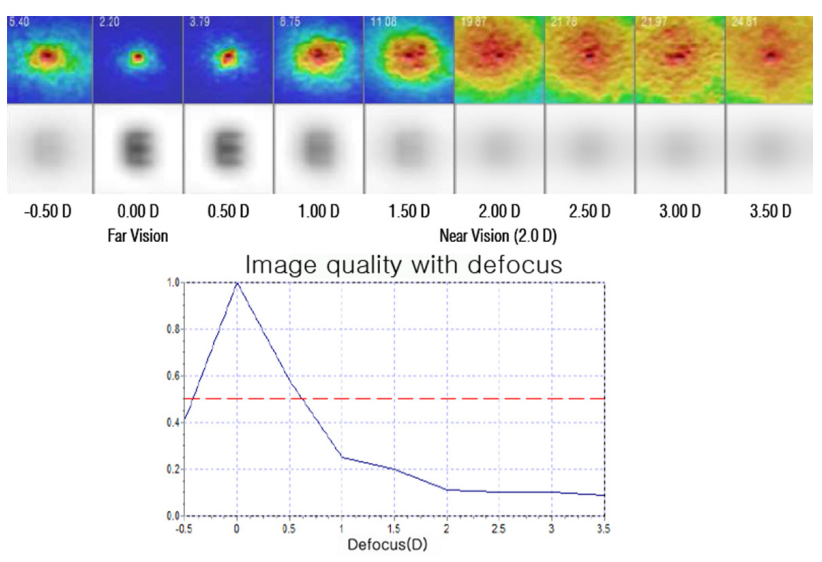

(a)

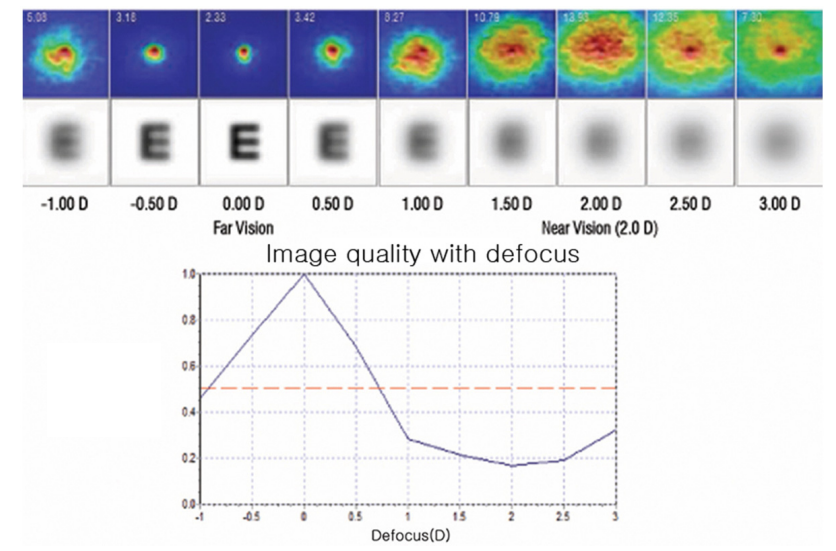

(b)

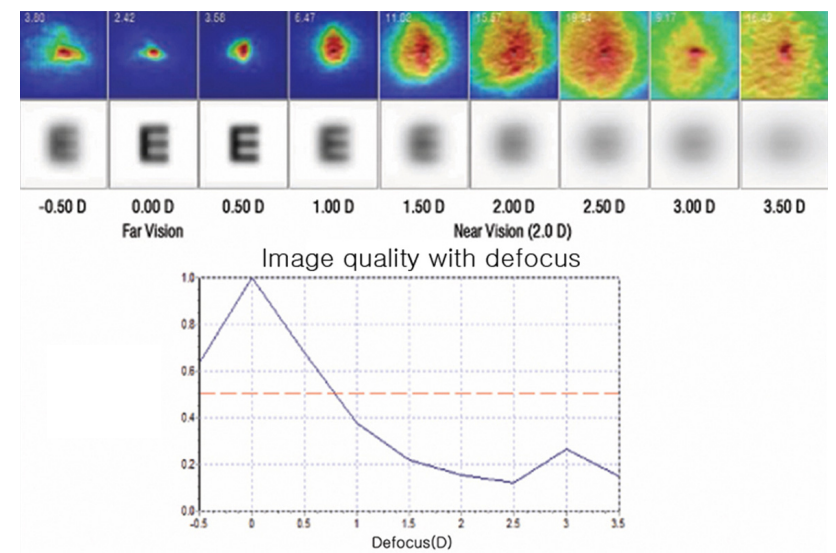

(c)

FIG. 3. Pseudo-accommodation curves of ReSTOR. (a) A monofocal curveof ReSTOR with $3 \mathrm{~mm}$ pupil; (b) A multifocal curve of ReSTOR with $3 \mathrm{~mm}$ pupil; (c) A multifocal curve of ReSTOR with $5 \mathrm{~mm}$ pupil.

and intermediate distance, but was better than that of IQ at very near distance.

\section{2. $5 \mathrm{~mm}$ Pupil}

With respect to MTF cut off, Strehl ratio, and PSF width at $50 \%$, the optical quality of ReSTOR decreased

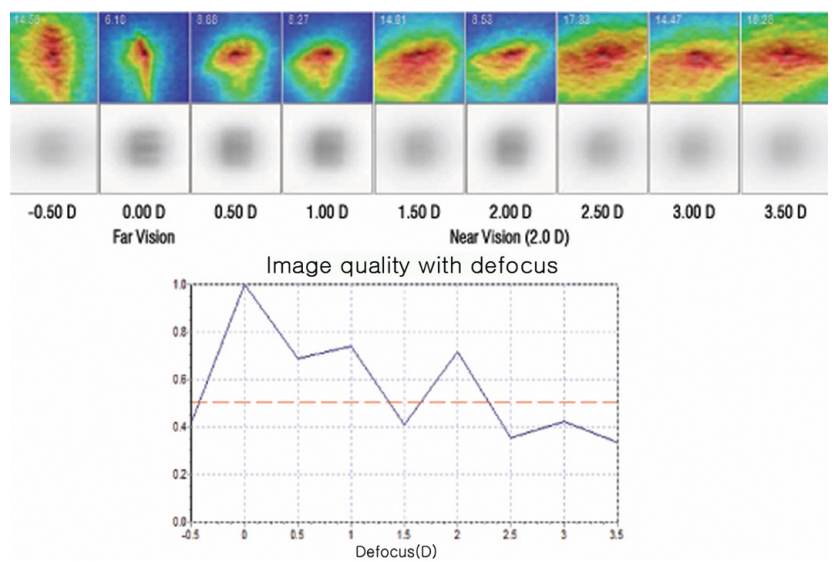

FIG. 4. A case mimicking multifocal function because of incomplete correction of cylindrical error.

from $0 \mathrm{D}$ to $-3.5 \mathrm{D}$ (Figs. 2(b), 2D, 2F). The optical quality of ReSTOR was worse than that of IQ at intermediate distance, with respect to PSF width at $50 \%$.

In the 'IOL accommodation curves' of the ReSTOR group, of the 11 curves for the $3 \mathrm{~mm}$ pupil, 3 showed monofocal curves (Fig. 3(a)) and 8 showed multifocalcurves (Fig. 3(b)). Of the 12 curves of the $5 \mathrm{~mm}$ pupil, 7 showed monofocal curves and 5 showed multifocalcurves (Fig. 3(c)). In the 'IOL accommodation curves' of the ReSTOR group, there was a case that showed a multifocalcurve for the $3 \mathrm{~mm}$ pupil and a monofocal curve for the $5 \mathrm{~mm}$ pupil (not shown here). Conversely, there was a case that showed a monofocal curve for the $3 \mathrm{~mm}$ pupil and a multifocalcurve for the $5 \mathrm{~mm}$ pupil (not shown here). In the 'IOL accommodation curves' of the IQ group (6 eyes), there were no multifocal curves for the $3 \mathrm{~mm}$ or $5 \mathrm{~mm}$ pupil and they showed only monofocal curves.

Figure 4 is an'IOL accommodation curve' of an IQ patient. Because of incomplete correction of the cylindrical error, the PSF was spread vertically at $-0.5 \mathrm{D}, 0 \mathrm{D}$ and horizontally at $+1.5 \mathrm{D}$. IOL accommodation curve of this IQ could be misrecognized as multifocal function.

\section{DISCUSSION}

Recently, Castillo-Go'mez et al. reported evaluation of image quality after implantation of 2 diffractive multifocal intraocular lens models using OQAS [10]. And Moreno et al. reported visual outcomes and optical performance of an apodized diffractive multifocal intraocular lens using OQAS [11]. However, they measured the optical quality only for far distance and did not measure the multifocal function of the IOL. In addition, they did not do a comparative analysis with other monofocal IOLs.

This is the first study that evaluated the multifocal function of ReSTOR as a diffractive multifocal IOL compared to the IQas a monofocal IOL with a double pass system (OQAS) that can objectively measure the optical quality in 
vivo.In this study, we could not find the statistically significant multifocal function of ReSTOR compared to IQ with respect to MTF cut off, Strehl ratio and PSF width at $50 \%$ (Table 2, 3). PSF width at 50\% (3mm pupil, very near distance) was the only parameter that shows the optical quality of ReSTOR at near or very near distance was better than that of IQ. However, becausethe MTF cut off and Strehl ratio of ReSTOR at that condition (3mm pupil, very near distance) was worse than those of IQ, it is questionable. If ReSTOR showed multifocal function by OQAS, for example, MTF cut off would decrease from 0 $\mathrm{D}$ and increase again from $-1.25 \mathrm{D}$ and show a second maximum at $-2.5 \mathrm{D}$ or $-3.5 \mathrm{D}$. But we could not find that kind of behavior of MTF, Strehl ratio and PSF width at $50 \%$ (Fig. 2).

We are not sure whether some 'IOL accommodation curves'of ReSTOR (Figs. 2(b), (c) really mean the multifocal function of ReSTOR or not. They are quite different from defocus curves in ReSTOR implantation patients [13-15]. We expected the second peak of pseudo-accommodation curvesat +2.5 Dbecause the SND1 model has the addition of $+3 \mathrm{D}(+2.5 \mathrm{D}$ at the spectacle plane). But, the multifocal curves of ReSTOR showed second peak at +3.0 $\mathrm{D}$ and over. Furthermore, we could not find the statistically significant multifocal function of ReSTOR compared to IQ with respect to MTF cut off, Strehl ratio and PSF width at $50 \%$ at all distances including very near distances $(-3.5 \mathrm{D})$.

In this study, the subjective near visual acuity of the ReSTOR group was better than that of the IQ group like in another study [1-6]. However, we could not prove this with objective tools such as OQAS. This issue was raised lately by Gatinel [12]. ReSTOR was designed as a diffractive multifocal IOL for visible light and OQAS uses infrared light as its light source; thus, it may be difficult for OQAS to measure the multifocal function of ReSTOR correctly, and the second peak in the pseudo-accommodation curves will be negligible. Furthermore, unlike real situations, the reflected light from the retina during OQAS measurement will develop diffraction at the diffraction rings of ReSTOR again as well as entering light into eyes. It might be one of the reasons which make OQAS unable to prove the multifocal function of ReSTOR.

The artificial pupil of OQAS will not adequately reflect the effect of the real pupil size. For real eyes, the pupil size for the incident light is important, because ReSTOR has diffraction rings at the central $3.6 \mathrm{~mm}$. However, in OQAS, the pupil size for the incident light is fixed and only the pupil size for the reflected light from retina can be changed.[10] This was also indicated as a problem by Gatinel.[12]In this study, we found a case that showed a multifocal curve for the $3 \mathrm{~mm}$ pupil and a monofocal curve for the $5 \mathrm{~mm}$ pupil. Conversely, there was a case that showed a monofocal curve for the $3 \mathrm{~mm}$ pupil and a multifocal curve for the $5 \mathrm{~mm}$ pupil.For large pupils, the depth of focus is shallow and it is possible that the multifocal function of the lens would be poor. On the other hand, since the diffractive rings are fully contributing, it is possible that the multifocal function would be better. For a small pupil, the depth of focus is deep and it is possible that a multifocal function would be better. On the other hand, only some diffractive rings contribute and it is possible that a multifocal function would be worse.

IQis a monofocal lens, contains yellow chromophores, and has an aspheric surface. Acrysof ReSTOR ${ }^{\circledR}$ is a diffractive multifocal IOL. It has a multifocal function by adding diffractive rings to Acrysof $\mathrm{IQ}^{\circledR}$. The SND1 model has 9 diffraction rings at the central $3.6 \mathrm{~mm}$ and has the addition of $+3 \mathrm{D}(+2.5 \mathrm{D}$ at the spectacle plane). SND3 has 16 diffraction rings at the central $3.6 \mathrm{~mm}$ and has the addition of $+4 \mathrm{D}(+3.2 \mathrm{D}$ at spectacle plane). We included only the patients with implantation of the SND1 model $(+2.5 \mathrm{D}$ at the spectacle plane) in this study. Therefore, we defined $-2.5 \mathrm{D}$ as near vision and defined $-1.25 \mathrm{D}$ as intermediate vision.

When we perform the OQAS measurement, it is very important to correct the cylindrical error by inserting a cylinder lens after auto-keratorefractometery. If the cylindrical error is not corrected for completely, the PSF is distorted vertically or horizontally. The IOL accommodation curve shows multipeaks and can be misrecognized to have a multifocal function (Fig. 4). If the PSF was distorted severely because of incomplete correction of the cylindrical error, the PSF quality was not good enough and the first peak of the 'IOL accommodation curve' was not located at $0 \mathrm{D}$.

\section{CONCLUSION}

In summary, we evaluated the multifocal function of ReSTOR as a diffractive multifocal IOL compared to the IQ as a monofocal IOL with the double pass system (OQAS), which can objectively measure the optical quality in vivo. It was impossible to prove the multifocal function of ReSTOR compared to the IQ by OQAS.

\section{ACKNOWLEGMENT}

This work was supported by the Technology Innovation Program funded by the Ministry of Trade, Industry \& Energy, Republic of Korea (10040121).

\section{REFERENCES}

1. H. Bi, Y. Cui, X. Ma, W. Cai, G. Wang, P. Ji, and X. Xie, "Early clinical evaluation of AcrySof ReSTOR multifocal intraocular lens for treatment of cataract," Ophthalmologica 222, 11-16 (2008).

2. J. F. Blaylock, Z. Si, and C. Vickers, "Visual and refractive status at different focal distances after implantation of the 
ReSTOR multifocal intraocular lens," J. Cataract Refract. Surg. 32, 1464-1473 (2006).

3. P. J. Chiam, J. H. Chan, R. K. Aggarwal, and S. Kasaby, "ReSTOR intraocular lens implantation in cataract surgery: Quality of vision," J. Cataract Refract. Surg. 32, 1459-1463 (2006).

4. B. Cochener, B. Arnould, M. Viala, A. R. de Climens, and G. Berdeaux, "Corrected and uncorrected near and distance vision with ReSTOR compared to monofocal intraocular lens implantation after cataract surgery: A pooled analysis," Ophthalmologica 223, 128-135 (2009).

5. C. E. Souza, C. Muccioli, E. S. Soriano, M. R. Chalita, F. Oliveira, L. L. Freitas, L. P. Meire, C. Tamaki, and R. Belfort, Jr., "Visual performance of AcrySof ReSTOR apodized diffractive IOL: A prospective comparative trial," Am. J. Ophthalmol. 141, 827-832 (2006)

6. E. M. Vingolo, P. Grenga, L. Iacobelli, and R. Grenga, "Visual acuity and contrast sensitivity: AcrySof ReSTOR apodized diffractive versus AcrySof SA60AT monofocal intraocular lenses," J. Cataract Refract. Surg. 33, 1244-1247 (2007).

7. M. J. Kim, L. Zheleznyak, S. Macrae, H. Tchah, and G. Yoon, "Objective evaluation of through-focus optical performance of presbyopia-correcting intraocular lenses using an optical bench system," J. Cataract Refract. Surg. 37, 1305-1312 (2011).

8. M. Vilaseca, A. Padilla, J. Pujol, J. C. Ondategui, P. Artal, J. L. Guell, "Optical quality one month after verisyse and Veriflex phakic IOL implantation and Zeiss MEL 80 LASIK for myopia from 5.00 to 16.50 diopters," J. Refract Surg. 25, 689-98 (2009).
9. M. Vilaseca, M. Arjona, J. Pujol, L Issolio, and J. L. Guell, "Optical quality of foldable monofocal intraocular lenses before and after injection: Comparative evaluation using a double-pass system," J. Cataract Refract. Surg. 35, 1415-23 (2009).

10. A. Castillo-Gomez, D. Carmona-Gonzalez, J. M. Martinezde-la-Casa, C. Palomino-Bautista, and J. Garcia-Feijoo, "Evaluation of image quality after implantation of 2 diffractive multifocal intraocular lens models," J. Cataract Refract. Surg. 35, 12441250 (2009).

11. L. J. Moreno, D. P. Pinero, J. L. Alio, A. Fimia, and A. B. Plaza, "Double-pass system analysis of the visual outcomes and optical performance of an apodized diffractive multifocal intraocular lens," J. Cataract Refract. Surg. 36, 2048-2055 (2010).

12. D. Gatinel, "Double pass-technique limitations for evaluation of optical performance after diffractive IOL implantation," J. Cataract Refract. Surg. 37, 621-622 (2011).

13. Q. Wang, G. Zhao, and W. Jia, "Visual quality after AcrySof IQ ReSTOR intraocular lens implantation in eyes with high myopia," Eur. J. Ophthalmol. 22, 168-174 (2012).

14. W. A. Maxwell, R. J. Cionni, R. P. Lehmann, and S. S. Modi, "Functional outcomes after bilateral implantation of apodized diffractive aspheric acrylic intraocular lenses with $\mathrm{a}+3.0$ or +4.0 diopter addition power randomized multicenter clinical study," J. Cataract Refract. Surg. 35, 2054-2061 (2009).

15. J. F. Alfonso, L. Fernandez-Vega, J. I. Blazquez, and R. Montes-Mico, "Visual function comparison of 2 aspheric multifocal intraocular lenses," J. Cataract Refract. Surg. 38, 242-248 (2012). 